\title{
Nanotechnology, equity and global health
}

\author{
When developing nanotechnology solutions for global health it is important to be mindful of the ethical, \\ environmental, socio-economical, cultural and legal aspects associated with their deployment.
}

\author{
Fabio Salamanca-Buentello and Abdallah S. Daar
}

$\mathrm{T}$ he COVID-19 pandemic has brought about devastation worldwide and has become one of the most challenging global health threats in modern history. Advances in nanotechnology have come to the rescue in the form of rapid diagnostic tests and, perhaps more importantly, swiftly developed vaccines against SARS-CoV-2. At the same time, numerous non-technical issues have complicated the deployment of these novel technologies and have brought to the fore the urgent need to incorporate ethical, environmental, economic, legal, social and cultural ( $E^{3} \mathrm{LSC}$ ) considerations in the development and implementation of nanotechnological solutions for global health challenges.

In this Commentary, we use the development and implementation of COVID-19 related nanovaccines as windows to the broader field of the application of nanotechnology to global health. We consider several $\mathrm{E}^{3} \mathrm{LSC}$ challenges related to the concept of 'nanoequity', and we suggest some strategies to address these challenges.

\section{Medical nanotechnologies for the developing world}

In 2005, we identified the ten nanotechnology applications most relevant to the developing world and correlated them with the Millennium Development Goals ${ }^{1}$. Crucially, three of these applications (disease diagnosis and screening, drug delivery systems and health monitoring) are critical for global health; several of the other categories in this list, such as water treatment and remediation, agricultural productivity enhancement, and air pollution and remediation, are directly relevant to addressing global health issues.

The wide range of nanomedical applications for global health can impact nearly every medical specialty and can improve the quality of life of people in low- and middle-income countries (LMICs), extending life expectancies, and reducing overall costs of healthcare ${ }^{2}$. The convergence of nanotechnology with other advanced technologies has catalysed the development of thermostable, single-dose, needle-free vaccines with increased shelf life that reduce required dosages and transportation costs; easy to use, highly sensitive and specific, robust, portable handheld point-of-care diagnostic systems; and novel delivery systems for the slow and targeted release of medications that improve specificity and decrease side effects ${ }^{2-4}$. Table 1 lists representative examples of nanotechnology-related projects funded by the Grand Challenges in Global Health Initiative (https://gcgh.grandchallenges. org/grants).

To meet the life-threatening challenges of the current pandemic, the scientific community has responded with unprecedented speed. Nanomedical advances have borne fruit as at least three COVID-19 vaccines ${ }^{5,6}$. The first two vaccines licenced for clinical use in humans (developed by Pfizer/BioNTech ${ }^{7}$ and Moderna ${ }^{8}$ ) use lipid nanoparticles to cage, stabilize and transport mRNA molecules, while the Novavax vaccine ${ }^{9}$ uses a proprietary recombinant protein nanoparticle technology platform to generate antigens derived from the SARS-CoV-2 spike protein. Future developments in nanotechnology could enable the addition of desirable features to these vaccines such as thermostability (ideal for places in LMICs with no adequate drug storage capabilities and distribution networks), needle-free single dose application and increased safety and efficacy ${ }^{2,5}$.

\section{'Nanoequity' and the E'LSC challenges However, addressing global health challenges is not simply a matter of identifying technical problems and developing technological solutions to overcome them. The excitement over novel technologies can hinder serious examination of the social and cultural contexts in which health care is delivered, a crucial component of the success of any health intervention. As is the case with other nanomedical technologies, the development and implementation of the COVID-19 nanovaccines have materialized in specific social and cultural settings and have demanded making complex moral decisions transcending the technical dimension of these advances. In particular, one of the most severe moral failures in the implementation of measures to control}

the current pandemic has been the lack of equity in the distribution and application of the COVID-19 nanovaccines. Not only has the roll-out of the vaccines favoured wealthy populations and countries, but industrialized nations have hoarded the pre-ordered doses of the vaccine to the detriment of the vast majority of people living in the developing world $^{10,11}$. As Dr Tedros Adhanom Ghebreyesus, Director-General of the World Health Organization (WHO), has stated, "The world is on the brink of a catastrophic moral failure and the price of this failure will be paid with lives and livelihoods in the world's poorest countries"12.

This is a glaring example of the lack of equity that can characterize the use of nanomedical advances to address global health issues. If we accept the premise that equity is critical for global health, and that it should be the guiding principle in the pursuit of improving the quality of life for people worldwide ${ }^{13}$, then this absence of 'nanoequity' is beyond unacceptable. It also creates numerous risks that threaten to destabilize the world. Misusing or limiting access to medical nanotechnologies could create a 'nano-divide' between wealthy and poor populations and countries, exacerbating existing differences in health outcomes and resource and power disparities ${ }^{4,14-17}$. Moreover, all aspects of medical nanotechnology research, development and innovation (RDI) may come under the control of powerful interests, and market forces may bias the development of medical nanoapplications and nanoproducts towards the wants of the wealthy and not towards the needs of the poor. If markets and commercial prospects, instead of local needs, drive medical nanotechnology RDI, risks (financial or otherwise) can be externalized onto vulnerable populations $s^{14,16,17}$.

Lack of equity in the development and implementation of medical nanotechnologies may manifest in other ways. For example, the COVID19 nanovaccines require storage at very low temperatures. But these potentially life-saving innovations may be rendered ineffective by the lack of operational 'cold chains' in many developing countries. Thus, medical nanotechnologies may be unsuitable 
Table 1 | Examples of nanotechnology-related projects in the Grand Challenges in Global Health Initiative

\begin{tabular}{|c|c|}
\hline $\begin{array}{l}\text { Healthcare } \\
\text { approach }\end{array}$ & Examples \\
\hline Prevention & $\begin{array}{l}\text { Condoms made of natural rubber latex/graphene composites or coated with } \\
\text { super-hydrophilic nanoparticles to improve durability, safety and appeal }{ }^{33} \text {. } \\
\text { Lipid-based nanoparticles containing iron, folic acid and iodine, integrated into } \\
\text { cosmetic pastes such as mehendi or kohl, commonly used by rural women } \\
\text { in LMICs, to deliver micronutrients transdermally to expectant mothers to } \\
\text { improve foetal health }{ }^{34} \text {. } \\
\text { Chitosan nanoparticles as carriers for gene-silencing small RNAs that, after } \\
\text { ingestion by larvae of malarial vectors, can kill them }{ }^{35} \text {. }\end{array}$ \\
\hline Vaccination & $\begin{array}{l}\text { Nasally administered aerosol hepatitis B vaccine based on dry powder or } \\
\text { poly(lactic-co-glycolic acid) (PLGA)/polyethylene glycol (PEG) nanoparticles } \\
\text { containing recombinant hepatitis B surface antigen }{ }^{36} \text {. } \\
\text { Intranasally administered sub-unit nanovaccine against brucellosis }{ }^{37} \text {. } \\
\text { Dermal nanopatches with microscopic silicon projections coated with a malaria } \\
\text { vaccine in dry form that target immunologically sensitive cells within the skin's } \\
\text { outer layers }{ }^{38} \text {. } \\
\text { Pollen-mimicking nanoparticles that penetrate the skin through hair follicles } \\
\text { and release vaccines upon contact with human sweat, targeting dendritic cells } \\
\text { that surround hair follicles }{ }^{39} \text {. } \\
\text { Anti-diarrhoeal biocompatible oral vaccine based on antigen-containing } \\
\text { nanoparticles generated by a genetically engineered common gut bacterium }{ }^{40} \text {. }\end{array}$ \\
\hline $\begin{array}{l}\text { Diagnostics and } \\
\text { monitoring }\end{array}$ & $\begin{array}{l}\text { Point-of-care, ultraportable, hand-held, robust, multiplex diagnostic systems } \\
\text { that detect different pathogens simultaneously using a single biological sample, } \\
\text { and are capable of delivering results rapidly as simple visual readouts }{ }^{41-44} \text {. } \\
\text { Ultraportable, battery-powered, handheld diagnostic nanodevices that } \\
\text { couple magnetic nanotechnology with an array of magnetically responsive } \\
\text { nanosensors for rapid, multiplex molecular diagnostic tests }{ }^{45} \text {. } \\
\text { Tuberculosis detection systems that use a sensing plaster containing non-toxic } \\
\text { nanomaterial-based sensors that detect volatile biomarkers }{ }^{46,47} \text {. } \\
\text { Wearable, self-healing, self-powered nanodevices for the non-invasive } \\
\text { detection of physiologic markers for continuous health monitoring }{ }^{48} \text {. }\end{array}$ \\
\hline Treatment & $\begin{array}{l}\text { High-density lipoprotein nanocarriers for short-interfering RNAs (siRNA) for the } \\
\text { treatment of preeclampsia }{ }^{49,50} \text {. } \\
\text { Photo-thermosensitive liposome-based nanocarriers that can release } \\
\text { medications transdermally via microneedles upon exposure to sunlight or } \\
\text { light-emitting diodes, eliminating toxic side effects and the need for multiple } \\
\text { administrations }{ }^{51} \text {. } \\
\text { Amphiphilic polyanhydride (AmPa) nanoparticles for single-dose delivery } \\
\text { and slow-release of medications that target filarial nematodes and their } \\
\text { endosymbiont bacteria Wolbachia }{ }^{52} \text {. } \\
\text { Multilayered skin patches with amoxicillin encapsulated in lipid nanoparticles } \\
\text { for continued transdermal release in children with pneumonia in low- and } \\
\text { middle-income countries }{ }^{53} \text {. }\end{array}$ \\
\hline
\end{tabular}

to LMICs if developers in high-income countries (HICs) fail to consider infrastructural barriers such as unreliable or non-existent clean water supplies, minimally trained personnel, poor leadership and a lack of coordination between different levels of the supply chain ${ }^{3,18}$. Nanomedical products designed for use in HICs may not function properly in LMICs due to humidity, heat or dust. Diagnostic systems requiring single-use cartridges only available through the equipment manufacturer, or needing costly regular maintenance and repair, may not be suitable to LMICs ${ }^{3}$. It stands to reason that medical nanotechnologies should be developed with awareness of the target context in which they will be used, even if doing so reduces their versatility. Incorporating local expertise in the design of these technologies, and encouraging their development within LMICs, would seem a reasonable way to address these challenges.

The promise of COVID-19 nanovaccines has been clouded by the fear ${ }^{19}$ that multinational pharmaceutical corporations will be reluctant to share their intellectual property rights with LMICs outside of the scope of strict commercial transactions. Human Rights Watch has called for urgent waivers of intellectual property rules for coronavirus vaccine development ${ }^{20}$. Aggressive patenting of nanomedical products by HICs, broad and restrictive patents and trade barriers risk consolidating ownership of medical nanotechnologies in the industrialized world. This could stifle domestic RDI in LMICs, reducing their ability to develop applications that could benefit vulnerable populations; it could also force developing countries to pay exorbitant fees for the use of nanotechnologies created and patented in the industrialized world ${ }^{4,16}$. Moreover, if most patents related to medical nanotechnology focus on medical conditions common in rich countries at the expense of neglected diseases prevalent in LMICs, vulnerable populations in these countries will be at a severe disadvantage to address their most pressing medical concerns ${ }^{4}$. To make matters worse, regulatory frameworks in LMICs may lack the nimbleness and flexibility that novel medical nanotechnologies demand. Given how devastating the pandemic has been for vulnerable populations in poor countries, there is a compelling argument for companies to reduce the stringency with which they enforce their intellectual property protection.

Inadequate sensitivity to the social and cultural dimensions of medical nanotechnologies may be particularly harmful to vulnerable individuals in LMICs. They may be used as experimental subjects for nanomedicine clinical trials, receiving none of the benefits of the research projects $^{21}$. Their ideas about the definitions and limits of identity, normalcy, disability, health and disease, and their conceptions about what is and is not part of the natural human body, may be disregarded in their government's pursuit of 'keeping up' with innovations in nanotechnology $y^{4}$ Rejection of unfamiliar novel nanomedical technologies due to clashes with local beliefs and experience may be compounded by fear of being tested or treated for stigmatized diseases such as HIV/AIDS ${ }^{18}$. Individuals in LMICs without access to nanobiomedical advances or without the desire to take advantage of such technologies may be discriminated against ${ }^{4}$.

LMICs could bear a disproportionate burden of the negative environmental effects of medical nanomaterials and nanoproducts, especially in the absence of proper evaluations of their complete life cycles. The developing world could become the dumping ground of unwanted, low-quality or potentially toxic medical nanoproducts from industrialized nations. Furthermore, healthcare workers in LMICs may be particularly vulnerable to the negative effects of medical nanotechnologies: they may 
be exposed to highly toxic nanomaterials, and they risk being replaced by advanced nanodevices ${ }^{4,22}$.

It is not surprising that many vulnerable populations in LMICs have reacted with fear and apprehension towards the COVID19 nanovaccines, especially in countries that have suffered the ruthlessness of colonialism. Inequities in the development and implementation of these and other medical nanotechnologies may kindle such mistrust and cause considerable damage to public health in LMICs. For example, absence of trust in diagnostic nanodevices may lead to lack of compliance with negative results and to the consequent adverse effects of unnecessary overmedication ${ }^{18}$.

\section{The possible ways forward}

The global community has the ethical responsibility to harness promising advances in nanomedicine to mitigate the most pressing health needs of vulnerable populations, especially in the developing world, while simultaneously preventing a nano-divide ${ }^{2,4}$. In the case of the COVID-19 vaccines, WHO has issued a call to action ${ }^{2}$ to all countries to work "... in solidarity - and in each of their best interests - to ensure that within the first 100 days of [2021], vaccination of health workers and older people [is] underway in all countries." Progress in this laudable goal is being tracked in real time (https://pandem-ic. com/vaccine-equity-tracker). Moreover, several developing countries have demanded that the World Trade Organization exempt member countries from enforcing some patents, trade secrets or pharmaceutical monopolies under the organization's agreement on trade-related intellectual property rights (TRIPs) ${ }^{24}$. This clamour has been strongly supported by the WHO Director General ${ }^{25}$.

Broad vaccination against COVID-19 worldwide could be fulfilled by supporting the Access to COVID-19 Tools (ACT) Accelerator ${ }^{26}$ and its vaccine pillar, COVAX (https://www.who.int/initiatives/ act-accelerator/covax). The ACT Accelerator is a ground-breaking global collaboration that aims to accelerate the development, production and equitable access to COVID19 tests, treatments and vaccines. COVAX, co-led by the Global Alliance for Vaccines and Immunisation (GAVI), the Coalition for Epidemic Preparedness Innovations (CEPI) and $\mathrm{WHO}$, is a global initiative aimed at equitable access to COVID-19 vaccines.

In the spirit of these initiatives, we present in Table 2 a list of strategies that can potentially address both the lack of 'nanoequity' in the development and implementation of medical

Table 2 | Strategies addressing 'nanoequity' risks and challenges in the development and implementation of medical nanotechnologies for global health

\begin{tabular}{ll} 
Areas of action & Strategies \\
\hline Infrastructure & Encourage domestic RDI in medical nanotechnologies in LMICs that can \\
help address their most pressing health needs. \\
Support strong collaboration and linkages among academia, government \\
and industry. \\
Strengthen the local nanotechnology industry sector. \\
Establish development and manufacturing operations in target countries. \\
Field test medical nanotechnologies to demonstrate their efficacy \\
and safety in local contexts, confirm their environmental and cultural \\
acceptability, evaluate their application by individuals with little or no \\
training, and ensure their compatibility with local policy and regulatory \\
frameworks.
\end{tabular}

Nanogovernance

Set priorities in LMICs by identifying resources, capabilities, limitations, existing knowledge gaps, potential niche areas, competitive advantages, and opportunities for strategic engagement.

Ensure the commitment of decision-makers in LMICs to secure long-term government support and funding for medical nanotechnologies. Combine strong national institutions that orchestrate all efforts in nanomedicine with decentralization of nanotechnology RDI.

Nanodiplomacy and international collaboration

Regulatory frameworks and intellectual property

Education and capacity building

Public engagement
Facilitate South-South and North-South multinational collaborations through nanotechnology RDI networks, always including developing countries as fully participating members.

Establish fair trade agreements between HICs and LMICs for the commercialization of medical nanotechnologies.

Convert 'brain drain' into 'brain recirculation' in LMICs.

Take advantage of highly educated diasporas, the communities of individuals from developing countries living and working in industrialized nations in academia, research or industry.

Prevent LMICs from becoming the dumping ground of nanowaste and potentially toxic nanoproducts from HICs.

Design regulatory frameworks in LMICs that are efficient, flexible, transparent, adaptable to local contexts and able to keep pace with advances in medical nanotechnologies.

Harmonize design and usage of nanomedical devices among different manufacturers to facilitate use and prevent the risk of confusion or improper application.

Use patent pools, patent clearinghouses and open-source approaches to balance the promotion of nanomedicine RDI in LMICs while still ensuring access and availability of nanomedical products useful for local health needs.

Encourage broad, rigorous, interdisciplinary education in nanotechnology at all levels of instruction.

Support and fund capacity building efforts that provide education and training in public health for nanotechnologists, and in nanotechnology and nanomedicine for public health professionals, clinicians and government decision-makers.

Favour medical nanotechnologies that enable task-shifting or task-sharing, the strategic redistribution of tasks among health workforce teams and personnel from highly trained health workers to those with shorter training or fewer qualifications.

Favour ease-of-use over versatility in the design of nanomedical devices targeted to LMICs, minimizing the need for technology-specific training.

Develop clear, consistent, transparent and socially- and culturally sensitive public engagement strategies that encourage ongoing, active and openminded dialogue among the general public in LMICs about the potential benefits, risks and costs of medical nanotechnologies. Acknowledge the legitimate expectations, fears and concerns of specific populations in LMICs about medical nanotechnologies.

Avoid top-down, manipulative 'nanomarketing' campaigns, as well as communication strategies based on irrational fears and misinformation. Ensure the participation of all relevant stakeholders in mapping the future of medical nanotechnologies in LMICs. 
nanotechnologies, and several related $\mathrm{E}^{3} \mathrm{LSC}$ challenges, with the aim of facilitating the judicious development and implementation of medical nanotechnologies in LMICs. Some of these strategies could be incorporated into our 2005 proposal of issuing a series of Grand Challenges in Nanotechnology $y^{1,4,27,28}$, now with an urgent focus on global health.

In summary, advances in nanomedicine have life-saving potential for vulnerable populations in the developing world. However, their design, development and implementation must be guided by equity as both a moral principle and a policy goal.

A broad search for examples of the successful, sustained and sustainable implementation in LMICs of medical nanotechnologies designed to address global health challenges led to several innovations that, while promising ${ }^{29-31}$, still have not completed the translation "from the lab to the village" 32 . In other words, their long-term, continued use in the developing world demands overcoming many of the $\mathrm{E}^{3} \mathrm{LSC}$ challenges mentioned in this commentary.

The successful application of nanomedical technologies demands serious attention to the social and cultural contexts in which they will be applied, and requires understanding that, as exciting as they may be, technological solutions are deeply embedded and influenced by the milieu from which they arise and whose systemic inequities they can perpetuate.

Fabio Salamanca-Buentello (D) $1{ }^{凶}$ and Abdallah S. Daar 2,3,4

${ }^{1}$ Bridgepoint Collaboratory for Research and Innovation, Lunenfeld-Tanenbaum Research Institute, Sinai Health, Toronto, Ontario, Canada. ${ }^{2}$ Department of Surgery, Temerty Faculty of Medicine, Toronto, Ontario (Emeritus), Canada.
${ }^{3}$ Department of Clinical Public Health, Dalla Lana School of Public Health, University of Toronto (Emeritus), Toronto, Canada. ${ }^{4}$ Stellenbosch Institute for Advanced Study, Stellenbosch, Western Cape, South Africa.

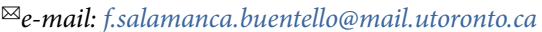

Published online: 29 March 2021

https://doi.org/10.1038/s41565-021-00899-z

\section{References}

1. Salamanca-Buentello, F. et al. PLoS Med. 2, e97 (2005).

2. Pautler, M. \& Brenner, S. Int. J. Nanomedicine 5, 803-809 (2010).

3. Abbas, J. J., Smith, B., Poluta, M. \& Velazquez-Berumen, A. Nanobiomedicine 4, 1-14 (2017).

4. Salamanca-Buentello, F. \& Daar, A. S. in Global Bioethics: The Impact of the UNESCO International Bioethics Committee (eds Moreno, J. D. et al.) 101-23 (Springer, 2016).

5. Chung, Y. H., Beiss, V., Fiering, S. N. \& Steinmetz, N. F. ACS Nano 14, 12522-12537 (2020).

6. Medhi, R., Srinoi, P., Ngo, N., Tran, H. \& Lee, T. R. ACS Appl. Nano Mater. 3, 8557-80 (2020)

7. Sahin, U. et al. Nature 586, 594-9 (2020).

8. Corbett, K. S. et al. N. Engl. J. Med. 383, 1544-55 (2020).

9. Tian, J. H. et al. Nat. Commun. 12, 372 (2021).

10. Mullard, A. How COVID vaccines are being divvied up around the world. Nature https://www.nature.com/articles/d41586-02003370-6 (2020)

11. Smith, M. J. \& Upshur, R. E. G. J. Bioeth. Inq. 17, 563-566 (2020).

12. WHO Director-General's opening remarks at the 148th session of the Executive Board. World Health Organization https://www.who.int/director-general/speeches/ detail/who-director-general-s-opening-remarks-at148th-session-of-the-executive-board (2021).

13. Eyawo, O. \& Viens, A. M. J. Bioeth. Inq. https://doi.org/10.1007/ s11673-020-10001-2 (2020).

14. Invernizzi, N. \& Foladori, G. Development 49, 114-118 (2006).

15. DePew, R. Nanotechnology in global health. In Proc. 40th Annual Northeast Bioengineering Conference (NEBEC) (Eds. Asthagiri, A. \& Carrier, R.) 1-2 (IEEE, 2014).

16. MacLurcan, D. C. Nanotechnology and global equality (Jenny Stanford Publishing, 2012).

17. Invernizzi, N., Foladori, G. \& Maclurcan, D. Sci. Technol. Soc. 13 123-48 (2008).

18. Miller, E. \& Sikes, H. D. Nanobiomedicine 2, 6-27 (2015)

19. Posner, G. Big pharma may pose an obstacle to vaccine development. The New York Times (2 March 2020); https://www.nytimes.com/2020/03/02/opinion/contributors/ pharma-vaccines.html

20. Urgently waive intellectual property rules for vaccine. Human Right Watch https://www.hrw.org/news/2020/12/10 urgently-waive-intellectual-property-rules-vaccine (2020).

21. Woodson, T. \& Rodriguez, V. Nanomedicine 14, 1745-1757 (2019).

22. Schummer, J. in Nanotechnologies, ethics, and politics (ed Ten Have, H.) 79-98 (UNESCO, 2007).
23. Call to action: vaccine equity declaration. World Health Organization https://www.who.int/campaigns/annual-theme/ year-of-health-and-care-workers-2021/vaccine-equitydeclaration (2021)

24. Want vaccines fast? Suspend intellectual property rights. The New York Times (7 December 2020); https://www.nytimes. com/2020/12/07/opinion/covid-vaccines-patents.html

25. Ghebreyesus, T. A. A 'me first' approach to vaccination won't defeat Covid. The Guardian (5 March 2021); https:// www.theguardian.com/commentisfree/2021/mar/05/ vaccination-covid-vaccines-rich-nations

26. The Access to COVID-19 Tools (ACT) accelerator. World Health Organization https://www.who.int/initiatives/ act-accelerator (2021).

27. Court, E. B., Salamanca-Buentello, F., Singer, P. A. \& Daar, A. S. in Nanotechnologies, ethics, and politics (ed. Ten Have, H.) 155-80 (UNESCO, 2007).

28. Singer, P., Daar, A., Salamanca-Buentello, F. \& Court, E. Georg. J. Int. Aff. 7, 129-37 (2006)

29. Brinkmann, A. et al. PLoS Negl. Trop. Dis. 11, e0006075 (2017).

30. Gardy, J. L. \& Loman, N. J. Nat. Rev. Genet. 19, 9-20 (2018).

31. Smith, C. et al. J. Clin. Microbiol. 59, e00583-20 (2021).

32. Daar, A. \& Singer, P. The Grandest Challenge: Taking Life-Saving Science from Lab to Village (Penguin Random House Canada, 2011).

33. Cooper, B. G. et al. R. Soc. Open Sci. 5, (2018).

34. Kapoor, M. S. et al. Sci. Rep. 8, 1-12 (2018).

35. Zhang, X. et al. J. Vis. Exp. 2015, e52523 (2015).

36. Muttil, P. et al. AAPS J. 12, 330-337 (2010).

37. Vyas, S., Dhoble, S., Ghodake, V. \& Patravale, V. Int. J. Pharm. X 2, 100053 (2020).

8. Pearson, F. E. et al. PLoS One 8, e67888 (2013).

39. Lehr, C.-M., Hansen, S., Schaefer, U. F. \& Guzman, C. A. Method for vaccination. WIPO patent WO/2011/138050 (2011).

40. Vohra, P. \& Blakely, G. W. Syst. Synth. Biol. 7, 73-78 (2013).

41. Lafleur, L. et al. Lab Chip 12, 1119-1127 (2012)

42. Lee, S. et al. Biosens. Bioelectron. 78, 290-299 (2016)

43. Derda, R. et al. PLoS Negl. Trop. Dis. 9, 1-16 (2015).

44. Choudhary, T., Rajamanickam, G. P. \& Dendukuri, D Lab Chip 15, 2064-2072 (2015).

45. Gaster, R. S., Hall, D. A. \& Wang, S. X. Lab Chip 11, 950-956 (2011).

46. Nakhleh, M. K. et al. Eur. Respir. J. 43, 1519-1522 (2014).

47. Konvalina, G. \& Haick, H. Acc. Chem. Res. 47, 66-76 (2014).

48. Wu, W. \& Haick, H. Adv. Mater. 30, el705024 (2018).

49. Osborn, M. F. \& Khvorova, A. Nucleic Acid Ther. 28, 128-136 (2018)

50. Schroeder, A., Levins, C. G., Cortez, C., Langer, R. \& Anderson, D. G. J. Intern. Med. 267, 9-21 (2010).

51. Mathiyazhakan, M., Wiraja, C. \& Xu, C. Nano-Micro Lett. 10, 10 (2018).

52. Binnebose, A. M. et al. PLoS Negl. Trop. Dis. 9, e0004173 (2015).

53. Khoshnevisan, K. et al. Carbohydr. Polym. 198, 131-141 (2018).

Competing interests

The authors declare no competing interests. 